\title{
Effect of emulsification method and particle size on the rate of in vivo oral bioavailability of kenaf (Hibiscus Cannabinus L.) seed oil
}

\begin{abstract}
Kenaf (Hibiscus cannabinus L.) seed oil-in-water nanoemulsions stabilized by complexation of beta-cyclodextrin with sodium caseinate and Tween 20 have been shown to have higher bioaccessibility of vitamin $\mathrm{E}$ and total phenolic content than nonemulsified kenaf seed oil in the previous in vitro gastrointestinal digestion study. However, its oral bioavailability was unknown. Therefore, the aim of this study was to evaluate the rate of in vivo oral bioavailability of kenaf seed oil-in-water nanoemulsions in comparison with nonemulsified kenaf seed oil and kenaf seed oil macroemulsions during the $180 \mathrm{~min}$ of gastrointestinal digestion. Kenaf seed oil macroemulsions were produced by using conventional method. Kenaf seed oil-in-water nanoemulsions had shown improvement in the rate of absorption. At $180 \mathrm{~min}$ of digestion time, the total $\alpha$-tocopherol bioavailability of kenaf seed oil nanoemulsions was increased by 1.7- and 1.4-fold, compared to kenaf seed oil and macroemulsion, respectively. Kenaf seed oil-in-water nanoemulsions were stable in considerably wide range of $\mathrm{pH}(>5$ and $<3)$, suggesting that it can be fortified into beverages within this $\mathrm{pH}$ range PRACTICAL APPLICATION: The production of kenaf seed oil-inwater nanoemulsions had provided a delivery system to encapsulate the kenaf seed oil, as well as enhanced the bioaccessibility and bioavailability of kenaf seed oil. Therefore, kenaf seed oil-in-water nanoemulsions exhibit a great potential application in nutraceutical fields.
\end{abstract}

Keyword: Bioavailability; Kenaf seed oil; Macroemulsion; Nanoemulsion; $\alpha$-tocopherol 